\title{
IMPORTANCE OF PHYSICAL HEALTH IN THE LONG-TERM MANAGEMENT OF BIPOLAR I DISORDERS
}

\section{De Hert}

University Psychiatric Centre Campus Kortenberg, Professor Psychiatry, Belgium

Bipolar I disorder is associated with a number of physical health concerns and a higher prevalence of eating, anxiety, and substance abuse disorders compared with the general population. In addition, patients are prone to exhibit risk factors for cardiovascular disease such as obesity, hypertension, hyperglycaemia, dyslipidaemia, and type 2 diabetes. ${ }^{1}$ Moreover, pharmacotherapy has been linked to metabolic abnormalities that may exacerbate the physical health risk factors and comorbidities prevalent in patients with bipolar disorder.

Choosing an initial treatment regimen that takes into account the significant physical health risks associated with bipolar disorder may minimise the subsequent common problem of polypharmacy and may reduce the side-effect burden associated with the disease. Regular monitoring of physical health in the months after initiating pharmacotherapy is essential in preventing and correctly managing comorbidities. Clinicians should gain a comprehensive insight into patient lifestyle choices and should, as part of preventative care, choose treatments according to individual patient risk profiles. If required, clinicians should make recommendations to specialists or facilities offering therapies to minimise key risk factors. ${ }^{2}$

This presentation will review the physical health risks associated with bipolar disorder and with chronic exposure to pharmacotherapy. Because practice guidelines recommend regular physical health monitoring in patients with bipolar disorder, this presentation will discuss approaches to incorporate regular monitoring in routine clinical practice.

\section{References}

1. De Hert $\mathrm{M}$ et al. Cardiovascular disease and diabetes in people with severe mental illness position statement from the European Psychiatric Association (EPA), supported by the European Association for the Study of Diabetes (EASD) and the European Society of Cardiology (ESC). European Psychiatry 2009;24:412-424.

National Institute for Health and Clinical Excellence. CG38 Bipolar disorder: NICE guideline. Available at: http://www.nice.org.uk/page.aspx?o=cg38niceguideline (Accessed January 2013) 